\title{
Pragmatic Interoperability: A Systematic Review of Published Definitions
}

\author{
Camlon H. Asuncion and Marten J. van Sinderen \\ Center for Telematics and Information Technology (CTIT), University of Twente \\ P.O. Box 217, 7500 AE Enschede, The Netherlands \\ $\{c \cdot h$.asuncion, m.j.vansinderen\} autwente.nl
}

\begin{abstract}
Enabling the interoperability between applications requires agreement in the format and meaning (syntax and semantics) of exchanged data including the ordering of message exchanges. However, today's researchers argue that these are not enough to achieve a complete, effective and meaningful collaboration - the use of data (pragmatics) is important as well. Pragmatic interoperability requires mutual understanding in the use of data between collaborating systems. However, we observe that the notion of pragmatic interoperability is still largely unsettled, as evidenced by the various proposed definitions and the lack of a canonical understanding. Therefore, our objective is to contribute to a more thorough understanding of this concept through a systematic review of published definitions. Our results show that, indeed, various interpretations of pragmatic interoperability exist. Categorizing the derivable concepts from these definitions, we see two broad groups: system level and business level. Within each of these individual levels, we see some degree of agreement among the definitions. However, comparing the definitions across these levels, we observe no general agreement. At the system level, pragmatic interoperability essentially means sharing the same understanding of the intended and actual use of exchanged system message in a given context. At the business level, pragmatic interoperability goes beyond service use by considering also the compatibility of business intentions, business rules, organizational policies, and the establishment and maintenance of trust and reputation mechanisms between collaborating business parties.
\end{abstract}

Keywords: pragmatic interoperability, definitions, systematic review.

\section{Introduction}

Since the beginning of the 1980s, research towards the interoperability of enterprise applications has been steadily increasing [2]. The continued emergence and advances in networking, computing technologies and standards have stimulated this interest. On the one hand, organizations are exploring interoperability to build partnerships that add value to their products and services, and help explore new business opportunities. On the other hand, these advances also provide opportunities for organizations to new enable partnerships in ways that were not previously possible [8]. 
Interoperability means allowing one system to perform the operation of another [2]. Until now, interoperability has been understood in a largely layered fashion. A meaningful interoperation between enterprises can be achieved fully if it exists in all layers simultaneously: inter-enterprise coordination, business process integration, semantic application integration, syntactical application integration, and physical integration [3].

Currently, however, there are also researchers who advocate the importance of interoperability at the pragmatic layer. Using results from our research, we tentatively define pragmatic interoperability as the compatibility between the intended versus the actual effect of message exchange [7]. Thus, at the message level, mere agreement between the meanings (or semantics) of exchanged data and the structure (or syntax) which codifies these messages are not enough to achieve complete, effective, and meaningful collaboration. How data is used (or pragmatics) is also important and must, therefore, be mutually understood between collaborating systems.

However, we observe that the definition of pragmatic interoperability is still largely unsettled. Unlike syntactic and semantic interoperability definitions, a variety of pragmatic interoperability definitions are currently proposed, and there seems to be a lack of a canonical understanding. We argue that solutions not founded on a common understanding of pragmatic interoperability may lead to incompatible solutions. As we are currently developing a solution, we find it imperative to first explore the concept of pragmatic interoperability.

We expect to contribute to this understanding through a systematic review of published definitions. Although we aim at achieving consensus in this area, with the support from both the industry and academia, it is not the objective of this paper to propose a single definition but to gather existing definitions and review their key differences and similarities. To the best of our knowledge, no such review has yet been made. We hope that this paper can serve as a starting point towards a possible improvement in the understanding and communication between individuals and organizations working in pragmatic interoperability research, and ultimately, to aid in developing future solutions.

The rest of the paper is structured as follows: Section 2 describes the background to the concepts behind interoperability and pragmatic interoperability. Section 3 describes the review process we used to systematically gather published definitions. Next, Section 4 presents the search and analysis results that compares key concepts, similarities and differences of the identified definitions. Section 5 provides some analysis and discussion. Finally, Section 6 presents our conclusion and future work.

\section{Background}

By way of background, we briefly introduce the key terminologies of interoperability and pragmatic interoperability.

Interoperability. Several definitions of interoperability exist. The IEEE defines it as: "the ability of two or more systems or components to exchange information and to use the information that has been exchanged"[10]. ISO defines it as "the capability to communicate, execute programs, or transfer data among various functional units in a 
manner that requires the user to have little or no knowledge of the unique characteristics of those units"[11]. The Open Group defines it as "the ability of systems to provide and receive services from other systems and to use the services so interchanged to enable them to operate effectively together"'[12]. Researchers in enterprise interoperability define it as: "the ability for two systems to understand one another and to use functionality of one another'"[2]. And, in the context of Service Oriented Architectures: "the ability of the software systems to use each other's software services"[7]. Summarizing, interoperability allows some form of interaction between two or more systems so as to achieve some goal without having to know the uniqueness of the interacting systems [7].

Pragmatic interoperability. At this stage, we briefly introduce pragmatic interoperability based on our own research so as to give some background information.

Merriam-Webster's dictionary defines pragmatics or pragmatism as to do, to act, or to be practical from the word's Greek etymology pragmatikos or pragma.

In Information Systems research, most studies that apply pragmatism seem to draw their theoretical foundation from the Theory of Signs (Semiotics) of Charles Morris [5] where he discusses human interpretation over (non-)linguistic signs. Morris sees Semiotics (in Greek: interpreter of signs) as that which is comprised of three basic components: syntactics (or syntax), semantics, and pragmatics. Syntax deals with the abstract study of signs and their formal relation to one another without regard to their meaning and use. Semantics reifies syntactic elements of signs by adding meaning but not use. Finally, pragmatics encapsulates both syntax and semantics for the purposeful use of signs [1]. Specifically in Morris' terms: syntax is that which acts as a sign (the sign vehicle), semantics is that which the sign refers to (the designatum), and pragmatics is the effect of the sign on the interpreter (the interpretant) [5, p.3].

Pragmatic principles in linguistic communication also apply to the interoperability of systems. In order to allow systems to interoperate, the communication between them must therefore take place achieved through message exchange. Messages contain data that represent the properties or values about the entities or phenomena of the message's subject domain (i.e. that part of the world that the message is about).

However, it is not always the case that collaborating systems have a common manner of codifying, understanding, and using the data that is exchanged. The difference can also be viewed in three layers: syntactic, semantic, and pragmatic.

- To ensure syntactic interoperability, collaborating systems should have a compatible way of structuring data during exchange; i.e., the manner in which data is be codified using a grammar or vocabulary is compatible.

- To ensure semantic interoperability, the meaning of the syntactic elements should be understood by collaborating systems; i.e.; they share the same meaning of the data in relation to the entity or phenomena it represents in the real world.

- Finally, to ensure pragmatic interoperability, message sent by a system causes the effect intended by that system; i.e., the intended effect of the message is understood by the collaborating systems. Pragmatic interoperability can only be achieved if systems are also syntactically and semantically interoperable [7]. 


\section{Review Process}

To allow for a rigorous search of definitions, we use the procedures for adopting systematic reviews proposed by Kitchenham [4]. A systematic review consists of a research protocol which details the rationale of the survey, research questions, search strategy, selection criteria, synthesis and analysis of the extracted data. Such a review procedure is appropriate for our purpose since, as Kitchenham argues, it summarizes existing evidence, identifies gaps in current research and areas for further investigation, and provides a background in which to position new research objectives. In our case, we summarize current evidence in pragmatic interoperability research by surveying their definitions and identifying gaps for further research through an analysis of their similarities and differences. Figure 1 describes the review process graphically.

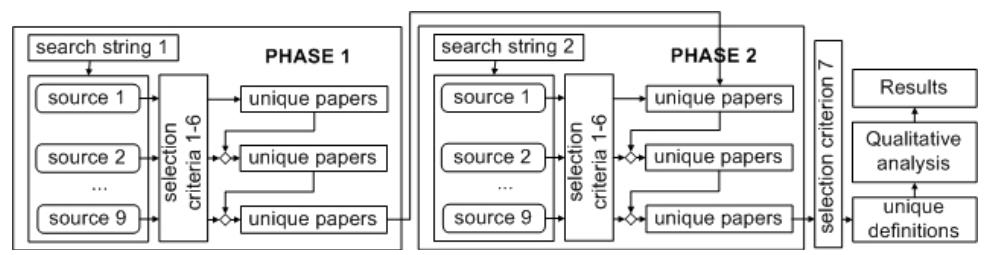

Fig. 1. Summary of the review process

We design the research to proceed in two phases. For each phase, we use a different search string (one broad and one narrow) to search for relevant papers. The search strings are used consistently over the same set of electronic indexing sources. From the hits returned by each source, we use a set of predefined selection criteria to manually identify papers that were both unique and relevant. To obtain only unique papers, we exclude a paper that has already been identified from a previously searched source regardless of the phase (i.e. they have the same paper title). Additionally, we manually looked into the references of selected papers which cite other authors to identify more definitions of pragmatic interoperability. We do this repeatedly - applying the same selection criteria - until no other referenced paper seem relevant. Finally, from the set of unique papers identified, we perform a qualitative analysis to draw key concepts and categories. The search was conducted from November 17, 2009 to January 31, 2010. One of us prepared the review protocol independently while the other reviewed and criticized it before the actual search began.

Research questions. Kitchenham[4] suggests that the structures of research questions should be divided into population, intervention and outcomes. We treat the population in our review as those pragmatic interoperability definitions proposed by various authors. Our intervention involves extraction, synthesis, and analysis of key concepts from these definitions. The outcome that is of interest to us is the similarities and differences between these definitions. Our research questions are thus: (i) What proposed definitions describing the notion of pragmatic interoperability can be found in the existing literature? (ii) What are the key concepts in these definitions? (iii) In what ways are the definitions similar or different? 
Search strategy. We conduct the search in two phases. The first phase searches for relevant papers as exhaustively as possible. We do this by using a search string whose main keywords included synonyms and word class variations. A preliminary search helped identify synonyms using the main keywords "pragmatic" and "interoperability", with additional help from a dictionary and thesaurus. For example, together with the main keyword "interoperability" we include its noun, adjective and verb forms; however, we do not add synonyms to the keyword "pragmatic" but add only its noun variation. The phase 1 search string is thus:

(pragmatic OR pragmatism) AND (interoperate OR
interoperability OR interoperable OR interoperation
OR integrate OR integration OR collaborate OR
COllaboration OR cooperate OR cooperation OR connect
OR connection OR communicate OR communication OR
exchange OR coalition)

The goal of the second phase is to make the search more restrictive, narrow and focused. The phase 2 search string is thus:

\section{pragmatic AND interoperability}

For each phase, we apply the search strings consistently over nine electronic indexing sources (searched in the following order): (i) Google Scholar (scholar.google.com), (ii) Scopus (www.scopus.com), (iii) ISI Web of Knowledge (apps.isiknowledge.com), (iv) CiteseerX (citeseerx.ist.psu.edu), (v) Compendex (www.engineeringvillage2.org), (vi) ScienceDirect (www.sciencedirect.com), (vii) IEEEXplore (ieeexplore.ieee.org), (viii) ACM Digital Library (portal.acm.org), and (ix) Springer Link (www.springerlink.com), sorting each search result by relevance.

Selection criteria. For each search phase, we devise and apply the same set of selection criteria to retrieve papers. The criteria include, in general: (i) limited to journals, conferences (proceedings), workshop papers, including technical reports, theses, and books or book chapters; (ii) written in English; (iii) regardless of publication date; (iv) within the computer science discipline; (v) in particular, it must explicitly contain text that defines (or attempts to define, propose, suggest, or describe) pragmatic interoperability found either in the abstract or body of the paper; (vi) proposed by original author(s); and (vii) in the case of similar definitions by the same (set of) original author(s), the most informative and descriptive definition (so not necessarily the latest). We used the first six criteria to extract candidate papers from the nine sources and the final criterion to arrive at the final set of definitions from the candidate papers for qualitative analysis.

Qualitative Analysis. From the total set of selected unique definitions, we analyze their differences and similarities using open coding - a component of the constant comparative method of analysis proposed by Strauss and Corbin [9]. Open coding is the process of analyzing data (which in our case are texts containing the definitions) by conceptualizing and categorizing them. Conceptualization requires breaking down, examining, comparing and labeling data according to some discrete happenings, ideas, events, or other phenomena. Categories group concepts which pertain to a similar phenomenon at a more abstract level. We use a qualitative analysis software 
called NVivo 8 [5] from QSR International to facilitate the coding process and to perform additional analysis. One of us performed the actual coding while the other reviewed and criticized the results.

\section{Results}

All in all, we identify 101 relevant and unique papers. From these, 43 papers are from the first phase, and 58 from the second. The 101 papers are unique in the sense that they do not have the same titles. However, it may be the case that they may have the same, or slightly similar, definitions from the same (set of) author(s). Thus, by applying the final selection criterion (c.f. Section 3), we arrive at the final set of unique definitions from 44 papers. These papers are the basis for the qualitative analysis using open coding that later followed. Table 1 shows the comparison between the key concepts derived from definitions of the selected 44 papers. The proposed categorizations of these concepts are discussed in Section 5.

Table 1. Summary of open coding analysis ${ }^{1}$

\begin{tabular}{|c|c|c|c|c|c|c|c|c|c|c|}
\hline \multirow[b]{2}{*}{ Authors } & \multirow[b]{2}{*}{$\underset{\nu}{\nexists ~}$} & \multicolumn{3}{|c|}{ System level } & \multicolumn{6}{|c|}{ Business level } \\
\hline & & 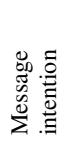 & 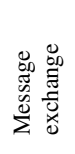 & 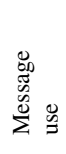 & 苍 & 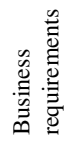 & 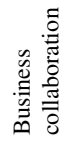 & 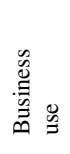 & 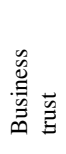 & 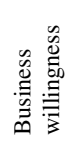 \\
\hline Pokraev [7] & 2009 & $\checkmark$ & $\checkmark$ & $\checkmark$ & & & & & & \\
\hline Roukolainen [13] & 2009 & & & & & $\checkmark$ & $\checkmark$ & & $\checkmark$ & $\checkmark$ \\
\hline Seo, et al. [14] & 2009 & $\checkmark$ & $\checkmark$ & $\checkmark$ & $\checkmark$ & & & & & \\
\hline Mingxin, et al. [15] & 2009 & $\checkmark$ & $\checkmark$ & $\checkmark$ & & & & & & \\
\hline Liu [16] & 2009 & $\checkmark$ & $\checkmark$ & $\checkmark$ & $\checkmark$ & & & & & \\
\hline Bravo, et al. [17] & 2009 & $\checkmark$ & $\checkmark$ & $\checkmark$ & $\checkmark$ & & & & & \\
\hline Sheping, et al. [18] & 2009 & $\checkmark$ & $\checkmark$ & $\checkmark$ & $\checkmark$ & & & & & \\
\hline Vilches-Blázquez, et al. [19] & 2009 & & & & $\checkmark$ & & & & & \\
\hline Tolk, et al. [20] & 2008 & & $\checkmark$ & $\checkmark$ & $\checkmark$ & & & & & \\
\hline Boxer, et al. [21] & 2008 & $\checkmark$ & $\checkmark$ & $\checkmark$ & $\checkmark$ & & & & & \\
\hline Leuchter, et al. [21] & 2008 & $\checkmark$ & $\checkmark$ & $\checkmark$ & & & & & & \\
\hline Dehmoobad, et al. [23] & 2008 & & & & & $\checkmark$ & $\checkmark$ & $\checkmark$ & & \\
\hline Ballari, et al. [24] & 2008 & $\checkmark$ & $\checkmark$ & $\checkmark$ & & & & & & \\
\hline Ruohomaa [25] & 2007 & & & & & $\checkmark$ & $\checkmark$ & & $\checkmark$ & $\checkmark$ \\
\hline de Moor [26] & 2007 & & & & $\checkmark$ & & & & & \\
\hline Elkin, et al. [27] & 2007 & & & & $\checkmark$ & & & & & \\
\hline Dagienè, et al. [28] & 2007 & $\checkmark$ & $\checkmark$ & $\checkmark$ & & & & & & \\
\hline Rukanova, et al. [29] & 2006 & & & & & $\checkmark$ & $\checkmark$ & $\checkmark$ & & \\
\hline Legner, et al. [30] & 2006 & & & & $\checkmark$ & $\checkmark$ & $\checkmark$ & $\checkmark$ & & \\
\hline Paterson, et al. [31] & 2006 & $\checkmark$ & $\checkmark$ & $\checkmark$ & & & & & & \\
\hline Tamani, et al. [32] & 2006 & $\checkmark$ & $\checkmark$ & $\checkmark$ & $\checkmark$ & & & & & \\
\hline Agerri, et al. [32] & 2005 & $\checkmark$ & $\checkmark$ & $\checkmark$ & $\checkmark$ & & & & & \\
\hline Wenzel, et al. [34] & 2005 & & & & $\checkmark$ & $\checkmark$ & $\checkmark$ & $\checkmark$ & & \\
\hline Schade, et al. [35] & 2005 & $\checkmark$ & $\checkmark$ & $\checkmark$ & $\checkmark$ & & & & & \\
\hline Artyshchev, et al. [36] & 2005 & $\checkmark$ & $\checkmark$ & $\checkmark$ & 冫 & & & & & \\
\hline Bazijanec, et al. [37] & 2005 & $\checkmark$ & $\checkmark$ & $\checkmark$ & & & & & & \\
\hline
\end{tabular}

${ }^{1}$ Due to lack of space, the list of selected definition and its summary are given in http://wwwhome.cs.utwente.nl/ asuncionch/research/pi/pi_definition_search_results.htm 
Table 1. (continued)

\begin{tabular}{|l|c|c|c|c|c|c|c|c|c|c|}
\hline Bentahar [38] & 2005 & $\checkmark$ & $\checkmark$ & $\checkmark$ & & & & & & \\
\hline Chun, et al. [39] & 2004 & $\checkmark$ & $\checkmark$ & $\checkmark$ & $\checkmark$ & & & & & \\
\hline Goossenaerts [40] & 2004 & $\checkmark$ & $\checkmark$ & & $\checkmark$ & & & & & \\
\hline Karasavvas, et al. [41] & 2004 & $\checkmark$ & $\checkmark$ & $\checkmark$ & & & & & & \\
\hline Hofmann [42] & 2004 & $\checkmark$ & $\checkmark$ & $\checkmark$ & $\checkmark$ & & & & & \\
\hline Zimmerman [43] & 2003 & $\checkmark$ & $\checkmark$ & $\checkmark$ & & & & & & \\
\hline Singh [44] & 2000 & $\checkmark$ & $\checkmark$ & & $\checkmark$ & & & & & \\
\hline Phillips, et al. [45] & 2002 & & & & $\checkmark$ & $\checkmark$ & $\checkmark$ & $\checkmark$ & & \\
\hline Euzenat [46] & 2001 & & & & $\checkmark$ & & & & & \\
\hline Huber, et al. [47] & 2000 & & $\checkmark$ & $\checkmark$ & & & & & & \\
\hline Labrou, et al. [48] & 1999 & $\checkmark$ & $\checkmark$ & $\checkmark$ & & & & & & \\
\hline Ingenerf [49] & 1999 & & $\checkmark$ & $\checkmark$ & $\checkmark$ & & & & & \\
\hline Wang, et al. [50] & 1999 & $\checkmark$ & $\checkmark$ & & & & & & $\checkmark$ & \\
\hline Bradshaw, et al. [51] & 1999 & $\checkmark$ & $\checkmark$ & $\checkmark$ & $\checkmark$ & & & & & \\
\hline Cerri [52] & 1999 & $\checkmark$ & $\checkmark$ & $\checkmark$ & & & & & & \\
\hline Gristock [53] & 1998 & & & & $\checkmark$ & & & & & \\
\hline Gitt [54] & 1989 & $\checkmark$ & $\checkmark$ & $\checkmark$ & & & & & & \\
\hline Werner [55] & 1988 & $\checkmark$ & $\checkmark$ & & $\checkmark$ & & & & & \\
\hline
\end{tabular}

\section{Discussion}

Although, in general, we see no agreement among the definitions, we observe that if the definitions are grouped broadly into two categories - system and business levels then we some reasonable agreement. By system level, we mean that the interaction is mostly between applications through the exchange of messages. By business level, we mean that the collaboration is mostly between organizations, business units, business processes, or even human actors [30]. Our results also show that much research emphasis has been given towards the system level and only a little at the business level (e.g. [13,23,25,29,30,34,45]).

At the system level, four key concepts consistently arise: message intention, message exchange, message use, and context.

- A message is sent with some intention. Some authors use the term 'goals' $[39,40]$, purpose [16], 'needs' [40], 'preferences' [18], 'desired actions' [42], or 'reasoning behind the message' [35]. A message intention contains what the sender expects the effect of the message will be or the intended use of data on the receiver.

- To realize the sender's message intention, the message must first be sent to the receiver in some automated way. This requires that message exchange must therefore take place. This is important as interoperability, by definition, requires communication between systems. We observe that all definitions at the system level consider this to be an important concept, whether stated explicitly or implicitly.

- Message use is about how a receiver interprets the intention of the communicated information on message receipt. Some authors call this generally as the effect the message has on the receiver (e.g. [7,31]). The actual effect of the message must thus be compatible with its desired intention. This demands from the receiver thorough understanding of the intention of the received message; i.e., the intended interpretation and use are clear (e.g. [14,16,17,18,20] among others). 
- The importance of context in the use, interpretation and understanding of the message is regarded by most authors as a core concept in pragmatic interoperability. It should not be the case that messages are used arbitrarily; a message has to be used in a certain context. The complete pragmatic meaning of a message varies, depending on the context in which it is used (e.g. $[20,16,14,30,32])$. Therefore, to achieve pragmatic interoperability, at least at the system level, the intention of the message and its use in a given context are understood by the collaborating parties; i.e., context is mutually shared [20]. Although some authors closely relate use and context (e.g. [20,16,14,32]), we separate them here as some authors are unclear as to their relation (e.g. [7,21,28,31]).

We notice also that there seems to be a lack of discussion and agreement as to what constitutes context in terms of its properties and dimensions in relation to pragmatic interoperability. Some authors do provide insights. Liu [16] says context is "where communication takes place. [It is] constantly and dynamically formed, deformed, configured and re-configured, and that [...] different behaviors can result [in] different results under different context [s]". Tolk [20] describes context as "both the state that the system is in at the time the [data] element is being employed, as well as a specification of the particular system process that will employ the [data] element. If any of these things change (either the system state, or the particular process), then the meaning of the element might be different".

Thus, summarizing, some authors believe that pragmatic interoperability, at the system level, is achieved if collaborating systems share the same intention of message use (e.g. [17,21]). Other authors emphasize the role of context beyond message use; i.e., pragmatic interoperability is achieved if collaborating systems share not only the same understanding of the intended use of data, but also the same context in which the message is (to be) applied (e.g. [14,20]). In a broader sense, we can consider that message use and the context where the use occurs together constitute the expected effect that must be both understood by collaborating systems (e.g. [7,16,31]).

At the business level, four main key concepts seem to arise consistently: business requirements, business collaboration, business use, and context.

- Business requirements encompass the business's autonomic intentions expressed through business rules, organizational policies [13], definition of responsibilities [23] and required business processes, specifications of the roles, and the definition of security services and authorization [34] needed for collaboration. The business requirements not only emphasize what the collaboration is about but also what the underlying business intentions are $[29,45]$.

- Like message exchange at the system level, business collaboration at the business level is also a prerequisite of pragmatic interoperability. This not only deals with continuous communication between business parties [32] but also entails negotiation [13,32] (e.g. expressed through collaboration contracts [13] including the execution and monitoring of responsibilities and agreements (such as through Service Level Agreements)[23]).

- Business use is an indication of how business parties use their shared services [23], how communicated information is received, interpreted [29], understood and used by partners [29,30], including who executes the communicated information [45]. 
- Similar to the system level, some argue that a shared understanding of background or context between the collaborating parties is also important to establish pragmatic interoperability at the business level. Context here includes the different professional, social, or cultural backgrounds of the collaborating business parties [32] relevant to the communication, or the sharing of the same physical space, same timeframe, and capabilities of collaborating parties [53].

- Aside from the four concepts outlined earlier, other authors also emphasize trust, reputation and willingness of collaborating partners as equally important prerequisites for pragmatic interoperability that must be established before and maintained during the collaboration [13,25,50]. Trust management looks at whether business parties trust one other enough to want to start a collaboration. Furthermore, the trust needed to start the collaboration may evolve over time and may be different for new parties joining. Business parties may also belong to different cultural norms and may follow different legislation that dictate how trust is to be established between them [25].

Thus, summarizing, pragmatic interoperability at the business level exists if there is compatibility between the business requirements of collaborating parties expressed through their business intentions, business rules, and organizational policies [13]. Collaborating parties should also have a shared understanding of the services they offer [30] and the context in which these services are to be used [32,29]. Beyond these, they should also establish beforehand and maintain during collaboration trust, willingness, and reputation-related issues [13,29].

\section{Conclusion and Future Work}

This paper explores the notion of pragmatic interoperability as it is understood today. As the understanding of the term is still largely unsettled, this paper aims to contribute to a more thorough understanding of the term through a systematic review of currently published definitions.

Our results show that, indeed, various interpretations of pragmatic interoperability exist. Categorizing the concepts from these definitions, we see two broad groups: system level and business level. Within these individual levels, we see some degree of agreement among the definitions. However, comparing the definitions across these levels, we observe no general agreement. At the system level, pragmatic interoperability involves sharing a common understanding and expectation in the use of data in a particular context (where context of use is much emphasized). At the business level, pragmatic interoperability entails a shared understanding of the use of services offered as applied in a given context. Beyond service use, collaborating parties should be compatible in terms of business intentions, business rules, organizational policies, including the establishment and maintenance of the trust and reputation mechanisms.

However, there still remain some key concepts that need to be further explored such as the notion of context in pragmatic interoperability. Although many authors argue favorably of its importance, we observe that there is still a lack of discussion and agreement especially at the business level where treatment is rudimentary. We 
ask, how should context be understood at either business or system level, and how does one influence the other particularly in pragmatic interoperability?

Additionally, the separation between the business level and system level needs a more thorough investigation in terms of their alignment. How can this alignment be achieved? What potential benefits and challenges can this alignment bring forth? In the end, we argue that to allow businesses to fully take advantage of pragmatic interoperability approaches, the system level and business level should be properly aligned.

At a much wider perspective, are the concepts of pragmatic interoperability presented in this paper currently addressed by solutions, approaches, frameworks or methodologies? If they do, to what extent are they able to do so? Are current languages or notations able to sufficiently express or model pragmatic interoperability requirements and solutions? If not, what new concepts have to be added to these languages or notations? These are just some of the important questions that will drive our future work in pragmatic interoperability research.

\section{References}

1. Allywood, J.: A Bird's Eye View of Pragmatics. In: 4th Scandinavian Conf. on Linguistics, pp. 145-159. Odense University Press (1978)

2. Chen, D., Doumeingts, G., Vernadat, F.: Architectures for Enterprise Integration and Interoperability: Past, Present and Future. Computers in Industry 59, 647-659 (2008)

3. Chen, D., Doumeingts, G.: European Initiatives to Develop Interoperability of Enterprise Applications - Basic Concepts, Framework and Roadmap. Annual Reviews in Control 27, 153-162 (2003)

4. Kitchenham, B.A.: Procedures for Performing Systematic Reviews. Technical Report TR/SE-0401, Keele University, and Technical Report 0400011T.1, NICTA (2004)

5. Morris, C.W.: Foundations of the Theory of Signs. University of Chicago Press, Chicago (1938)

6. NVivo v8, QSR International, http://www.qsrinternational.com/products_nvivo.aspx

7. Pokraev, S.V.: Model-Driven Semantic Integration of Service-Oriented Applications. PhD thesis, University of Twente (2009)

8. van Sinderen, M.J.: Challenges and Solutions in Enterprise Computing. Enterprise Information Systems 2, 341-346 (2008)

9. Strauss, A., Corbin, J.M.: Basics of Qualitative Research: Techniques and Procedures for Developing Grounded Theory. Thousand Oaks, California (1998)

10. Institute of Electrical and Electronics Engineers (IEEE): IEEE Standard Computer Dictionary: A Compilation of IEEE Standard Computer Glossaries (1990)

11. ISO/IEC 2382-1:1993 Information Technology - Vocabulary - Part 1: Fundamental terms. International Organization for Standardization, ISO (1993)

12. The Open Group, http://www.opengrouporg/architecture/togaf8doc/arch/chap36.html

13. Roukolainen, T.: Modeling Framework for Interoperability Management for Collaborative Computing Environments. Licentiate Thesis, University of Helsinki (2009)

14. Seo, C., Zeigler, B.P.: DEVS Namespace for Interoperable DEVS/SOA. In: 2009 Winter Simulation Conf., pp. 1311-1322 (2009)

15. Mingxin, G., Lily, S.: Dynamic Convergence of Business and IT Systems Through Enterprise Isomorphic Architecture. In: 2009 Int. Conf. on Information Technology and Computer Science, vol. 1, pp. 366-369. IEEE Computer Society, Los Alamitos (2009) 
16. Liu, K.: Pragmatic Computing - Semiotic Perspective to Web Services. In: 4th Int. Conf. on E-business and Telecommunications, vol. 23, pp. 3-15. Springer, Berlin (2009)

17. Bravo, M., Alvarado, M.: On the Pragmatic Similarity Between Agent Communication Protocols: Modeling and Measuring. In: On the Move to Meaningful Internet Systems: OTM 2008 Workshops, pp. 128-137. Springer, Berlin (2009)

18. Sheping, Z., Zengzhi, L., Juanli, W.: Negotiation Between Requester and Provider Based on Pragmatics Web Service. In: 2009 WRI Int. Conf. on Communications and Mobile Computing, vol. 3, pp. 583-587. IEEE Computer Society, Los Alamitos (2009)

19. Vilches-Blázquez, L.M., Corcho, O.: A Heuristic Approach to Generate Good-Quality Linked Data About Hydrography. In: 20th Int. Workshop on Database and Expert Systems Application, Linz, Austria, pp. 99-103. IEEE Computer Society, Los Alamitos (2009)

20. Tolk, A., Turnitsa, C., Diallo, S.: Implied Ontological Representation within the Levels of Conceptual Interoperability Model. Intelligent Decision Technologies 2, 3-19 (2008)

21. Boxer, P., Morris, E., Anderson, W., Cohen, B.: Systems-of-Systems Engineering and the Pragmatics of Demand. In: 2nd Annual IEEE Systems Conf., Canada (2008)

22. Leuchter, S., Mühlenberg, D., Schönbein, R.: Agent-Based Web for Information Fusion in Military Intelligence, Surveillance, and Reconnaissance. In: 2008 IEEE Int. Conf. Systems, Man and Cybernetics, pp. 3732-3737. IEEE Computer Society, Los Alamitos (2008)

23. Dehmoobad, A., Sartipi, K.: Minimized Domain Knowledge for SOA-Based Interoperability. In: IEEE Asia-Pacific Services Computing Conf., pp. 500-506 (2008)

24. Ballari, D., Manso-Callejo, M.A., Wachowicz, M.: The Interoperability of Wireless Sensor Networks. In: Sensors 2008, vol. 9, pp. 3635-3651 (2008)

25. Ruohomaa, S.: Trust Management for Inter-enterprise Collaborations. In: Web Proceedings of the I-ESA 2007 Doctoral Symposium, Portugal (2007)

26. de Moor, A.: The Pragmatic Evaluation of Tool System Interoperability. In: 2nd Conceptual Structures Tool Interoperability Workshop, pp. 1-19 (2007)

27. Elkin, P.L., Froehling, D., Bauer, B.A., Wahner-Roedler, D., Rosenbloom, S.T., Bailey, K., Brown, S.H.: Aequus Communis Sententia: Defining Levels of Interoperability. Studies in Health Technology and Informatics 129, 725-729 (2007)

28. Dagienè, V., Kurilovas, E.: Design of Lithuanian Digital Library of Educational Resources and Services: The Problem of interoperability. Information Technologies and Control 36, 402-411 (2007)

29. Rukanova, B., van Slooten, K., Stegwee, R.A.: Towards a Meta-model for Describing Communication: How to Address Interoperability on a Pragmatic Level. In: Enterprise Information Systems VI, pp. 203-210 (2006)

30. Legner, C., Wende, K.: Business Interoperability Framework, http: / /www.alexandria.unisg.ch/EXPORT/DL/39432.pdf

31. Paterson, G.I., Soroka, S.D.: Formative Evaluation of the Clinical Pragmatic Attributes of Components Chosen for a Boundary Infostructure. In: 11th Int. Symp. on Health Information Management Research (2006)

32. Tamani, E., Evripidou, P.: A Pragmatic and Pervasive Methodology to Web Service Discovery. In: OTM 2008 Workshops, pp. 1285-1294. Springer, Berlin (2006)

33. Agerri, R., Alonso, E.: Semantics and Pragmatics for Agent Communication. In: 12th Portuguese Conf. on Artificial Intelligence, pp. 524-535. Springer, Berlin (2005)

34. Wenzel, S., Jessen, U., Bernhard, J.: Classifications and Conventions Structure the Handling of Models within the Digital Factory. Computers in Ind. 56, 334-346 (2005)

35. Schade, U.: Towards the Edge and Beyond: The Role of Interoperability. In: 10th International Command and Control Research and Technology Symposium (2005) 
36. Artyshchev, S., Weigand, H.: Contract-based Interoperability for e-Business Transactions. In: 2005 Interoperability of Enterprise Software and Applications, pp. 173-184. Springer, London (2005)

37. Bazijanec, B., Zaha, J.M., Albani, A., Turowski, K.: Establishing Interoperability of Coordination Protocols in Ad Hoc Inter-Organizational Collaborations. In: 1st INTEROPESA 2005, pp. 126-136 (2005)

38. Bentahar, J.: A Pragmatic and Semantic Unified Framework for Agent Communication. PhD Thesis, Laval University, Canada (2005)

39. Chun, S.A., Lee, Y., Geller, J.: Ontological and Pragmatic Knowledge Management for Web Service Composition. In: 9th Int. Conf. on Database Systems for Advanced Applications, pp. 365-373. Springer, Heidelberg (2004)

40. Goossenaerts, J.: Interoperability in the Model Accelerated Society. In: Presentation at the eChallenges Conf., Austria (2004)

41. Karasavvas, K.A., Baldock, R., Burger, A.: Bioinformatics Integration and Agent Technology. J. Biomed. Inform. 37, 205-219 (2004)

42. Hofmann, M.A.: Challenges of Model Interoperation in Military Simulations. Simulation 80, 659-667 (2004)

43. Zimmermann, B.: Integrated Army Modeling and Simulation Data Network. Paper \#08, NATO Report RTO-MP-123: C3I and M\&S Interoperability, Turkey (2003)

44. Singh, M.P.: A Social Semantics for Agent Communication Languages. In: Issues in Agent Communication, pp. 31-45. Springer, Berlin (2000)

45. Phillips Jr., C.E., Ting, T.C., Demurjian, S.A.: Information Sharing and Security in Dynamic Coalitions. In: 7th ACM Sym. on Access Control Models and Technologies, Monterey, pp. 87-96 (2002)

46. Euzenat, J.: Towards a Principled Approach to Semantic Interoperability. In: Workshop on Ontologies and Information Sharing (2001)

47. Huber, T., Alt, R., Österle, H.: Templates - Instruments for Standardizing ERP Systems. In: 33rd Hawaii Int. Conf. on Science Systems, Maui, Hawaii (2000)

48. Labrou, Y., Finin, T.: History, State of the Art and Challenges for Agent Communication Languages. INFORMATIK - Zeitschrift der schweizerischen Informatik organisationen 7, 17-24 (1999)

49. Ingenerf, J.: Telemedicine and Terminology: Different Needs of Context Information. IEEE Trans. Inf. Technol. Biomed. 3, 92-100 (1999)

50. Wang, I., Liu, C., Conradi, R.: A Multi-Agent Architecture for Cooperative Software Engineering. In: 11th Int. Conf. on Software Engineering and Knowledge Engineering, Germany, pp. 1-22 (1999)

51. Bradshaw, J.M., Greaves, M., Holmback, H., Karygiannis, T., Jansen, W., et al.: Agent for the Masses? IEEE Intelligent Systems, 53-63 (1999)

52. Cerri, S.A.: Shifting the Focus from Control to Communication: the STReams OBjects Environments Model of Communicating Agents. In: Collaboration between Human and Artificial Societies, Coordination and Agent-Based Distributed Computing, pp. 74-101. Springer, Berlin (1999)

53. Gristock, J.J.: Organisational Virtuality: A Conceptual Framework for Communication in Shared Virtual Environments. In: MIT Media Lab/University College London workshop Presence in Shared Virtual Environments, Ipswich (1998)

54. Gitt, W.: Information: The 3rd Fundamental Quantity. Siemens Rev. 6, 36-41 (1989)

55. Werner, E.: Toward a Theory of Communication and Cooperation for Multi-Agent Planning. In: 2nd Conf. Theoretical Aspects of Reasoning About Knowledge, pp. 129-144 (1988) 